\title{
New Discovery on Planck Units and Physical Dimension in Cosmic Continuum Theory
}

\author{
Xijia Wang \\ College of Mathematics and Statistics, Hunan Normal University, Changsha, China \\ Email: xijiawang@hunnu.edu.cn
}

How to cite this paper: Wang, X.J. (2018) New Discovery on Planck Units and Physical Dimension in Cosmic Continuum Theory. Journal of Modern Physics, 9, 2391-2401. https://doi.org/10.4236/jmp.2018.914153

Received: November 6, 2018

Accepted: December 4, 2018

Published: December 7, 2018

Copyright $\odot 2018$ by author and Scientific Research Publishing Inc. This work is licensed under the Creative Commons Attribution International License (CC BY 4.0).

http://creativecommons.org/licenses/by/4.0/ (c) (i) Open Access

\begin{abstract}
In 1899, Max Planck integrated the Planck constant $h$ with the gravitational constant $G$ and the speed of light $c$, discovered a set of physical constants, and created Planck Units System. Since 20th century, the development of physics made the gravitational constant, the speed of light, and the Planck constant the most important fundamental constants of physics representing classical theory, relativity, and quantum theory, respectively. Now, the Planck Units have been given new physical meanings, revealing the mysteries of many physical boundaries. However, more than 100 years have passed, Planck Units System not only failed to get rid of the incompatibility between the basic theories of physics, but also cannot surpass the limitations of existing physics theories. In Cosmic Continuum Theory, physical dimensions can be transformed under the principle of equivalence. Planck units system not only integrates into the axiom system of Cosmic Continuum Theory, but also establishes a benchmark for the unity of physical dimensions. The introduction of the abstract physical dimensions "JX" and "XJ" makes the physical dimension of existence quantity and dimension quantity unified respectively.
\end{abstract}

\section{Keywords}

Cosmic Continuum, Axiomatic Physics, Fundamental Constants of Physics, Gravitational Redshift, Planck Units, Dimensional Analysis

\section{Introduction}

The fundamental constants of physics are the passwords of the universe and important scientific discoveries. Among all the physical constants, the well-known gravitational constant $G$, speed of light $c$, and Planck constant $h$ are the three most fundamental constants of physics. These three fundamental con- 
stants of physics represent the different epoch-making basic theory of physics respectively. The gravitational constant $G$ represents classical theory, the speed of light $c$ represents relativity, and the Planck constant $h$ represents quantum theory.

However, because these three basic theories of physics have different logical preconditions, they are regarded as three different cosmologies. There are disputes between high speed and low speed, macroscopic and microscopic, continuous and discrete between the three parties, and no consensus has been reached so far.

Cosmic Continuum Theory is an axiomatized physics system built on the mathematical continuum model. In Cosmic Continuum Theory, the universe is a continuum consisting of an existence continuum and an existing dimension continuum. The existence continuum is composed of mass bodies, energy bodies and dark mass bodies. The existing dimension continuum is composed of space, time and dark space. Mass, energy and dark mass are collectively called the existence quantity, and the quantity of space, time and dark space is called the dimension quantity [1] [2] [3].

This theory holds that a fundamental constant of physics should not only serve a certain physical basic theory. As cosmic codes of physics that gradually discovering in the course of continuous advancement, they are human's basic understanding of the natural world and should be an important basis for the unity of physics.

It was Max Planck himself who tried to unify fundamental constants of physics and achieved remarkable success. In 1899, he combined the Planck constant $h$ with the gravitational constant $G$ and the speed of light $c$, and found a series of physical constants. These physical constants are the so-called Planck units, and the following four constants are related to the existence quantity and the dimension quantity [4].

1) Planck mass: $m_{p}=\sqrt{h c / G} \approx 2.17651 \times 10^{-8} \quad(\mathrm{~kg})$.

2) Planck energy: $E_{p}=\sqrt{h c^{5} / G} \approx 1.9561 \times 10^{9} \quad(\mathrm{~J})$.

3) Planck time: $t_{p}=\sqrt{h G / c^{5}} \approx 5.39106 \times 10^{-44}$ (s).

4) Planck length: $l_{p}=\sqrt{h G / c^{3}} \approx 1.61619 \times 10^{-35}(\mathrm{~m})$.

They are given corresponding physical meanings, such as: the mass of the ground state particles cannot be greater than the Plank mass; the energy cannot be greater than or equal to the Planck energy, otherwise it will collapse into a black hole; Planck time is an observable event Minimum process time; Planck length is a measure of Plank's quality black hole, unable to distinguish events within a distance less than Planck length; unable to describe events occurring within Planck time when the universe was born, etc. However, the Planck Unit cannot resolve the logical contradiction with the continuous space-time of the theory of relativity, because the space-time structure derived from Planck's length and Planck's time is discontinuous. Even for quantum field theory, Planck length and point particle models are in conflict [5]-[21]. 


\section{The Physical Boundaries and Their Conversion of Planck Units System}

In the following, we include the Planck units in the axiom system of Cosmic Continuum Theory for discussion.

\subsection{Fundamental Concepts and Lemmas}

This article uses the fundamental concepts and axioms system in [1]. The following lemmas are cited in [1], these lemmas will be used in later proofs.

Lemma 1: The existence quantity has its elementary unit $e_{\min }$ (Proof see [1]).

This lemma is equivalent to the quantification hypothesis.

Lemma 2: The interaction force between the initial fields of the existences $Z_{1}$ and $Z_{2}$ is: $f \sim Z_{1} Z_{2} / r^{2}$, and the action direction is along the line connecting $Z_{1}$ and $Z_{2}$, where, $r$ is the distance between $Z_{1}$ and $Z_{2}$ (Proof see [1]).

This lemma contains the law of universal gravitation. When the existence is a mass body, the theorem is the law of universal gravitation.

Lemma 3: An existence has a maximum speed and the speed of an energy body is the maximum speed (Proof see [1]).

This lemma includes the speed of light postulate.

Lemma 4: An existence has a maximum frequency $v_{\max }$ (Proof see [1]).

Lemma 5: When the particle reaches its maximum speed, it will be converted to a quantum; when the particle or quantum reaches its maximum frequency, it will be transformed into dark particle (Proof see [1]).

Lemma 6: The existence quantities of mass $m$ and energy $E=m c^{2}$ are equivalent: $m \equiv E$. “”” is the "equivalent" symbol (Proof see [1]).

This lemmas contain mass-energy relation $E=m c^{2}$.

Lemma 7: The dimension quantity of 1-second of time is equivalent to that of $c$-kilometer space: 1 second $\equiv c \mathrm{~km}$, where, $c$ indicates the speed of light (Proof see [1]).

Lemma 8: In a cosmic system A, the elementary particle, elementary quantum and elementary dark particle have equivalent inertia size: $m_{\min } \equiv q_{\min } \equiv d_{\min }$ (Axiom in [1]).

Lemma 9: A dimension quantity has its elementary units $w_{\min }$, and the dimension quantities of elementary space quantity $s_{\min }$, elementary time quantity $t_{\min }$ and elementary dark space quantity $w_{\min }$ are equivalent:

$w_{\min } \equiv s_{\min } \equiv t_{\min } \equiv g_{\min } \quad$ (Proof see [1])

Lemma 10: The existences are coupled with each other by energy, And in the presence of different structural levels, by the corresponding quantum from the role of convergence. If there is a structure at the structural level of the quantum of $q$ connection, $e$ the corresponding amount of existence, the corresponding structure of the particle $m$, dark particle $d$ is also the amount of $e$ : $m \equiv d \equiv q \equiv e$ (Axiom in [1]).

Lemma 11: There are only three basic forms of existence: particle, quantum, 
and dark particle (Axiom in [1]).

Lemma 12: The interaction force between the initial fields of the existences $Z_{1}$ and $Z_{2}$ is: $f \sim Z_{1} Z_{2} / r^{2}$, and the action direction is along the line connecting $Z_{1}$ and $Z_{2}$, where, $r$ is the distance between $Z_{1}$ and $Z_{2}$ (Proof see [1]).

\subsection{Derivation}

\section{1) Planck mass $m_{p}$}

According to Lemma 2, the gravitational potential energy of two existing bodies with a distance of $r$ and a mass of $m$ is $G m^{2} / r$, where $G$ is the gravitational constant.

Let the vibration period of mass body $m$ be $t$, then its frequency is:

$$
v=1 / t
$$

Also set its speed to $V$, then its movement distance in a period $t$ is:

$$
r=V t
$$

The search for Planck unit is to define the boundaries of physical events. According to Lemma 3, the velocity boundary of the microscopic particles is the speed of light $c$, so the change in the gravitational potential energy between the two bodies of Planck mass $m_{p}$ in one cycle $t$ should be:

$$
\lim _{V \rightarrow c} G m_{p}^{2} /(V t)=G m_{p}^{2} /(c t)
$$

According to Lemma 1, the quantification hypothesis, we get:

$$
G m_{p}^{2} /(c t)=h v
$$

Substituting (1) into (4) yields:

$$
G m_{p}^{2} /(c t)=h / t
$$

From the formula (5), we can get the Planck mass $m_{p}$ :

$$
m_{p}=\sqrt{h c / G}
$$

2) Planck energy $E_{p}$

Planck energy $E_{p}$ can be obtained from Lemma 6:

$$
E_{p}=m_{p} c^{2}
$$

Substituting (6) into (7)) gives Planck energy $E_{p}$ :

$$
E_{p}=\sqrt{h c^{5} / G}
$$

\section{3) Planck time $t_{p}$}

According to Lemma 1, the Planck time $t_{p}$ can be found by:

$$
E_{p}=h v_{p}
$$

where $v_{p}$ is the frequency of the Planck time $t_{p}$ period:

$$
v_{p}=1 / t_{p}
$$

Substituting (10) into (9) yields: 


$$
E_{p}=h / t_{p}
$$

Substituting (8) into (11) yields:

$$
\sqrt{h c^{5} / G}=h / t_{p}
$$

Obtain $t_{p}$ from (12):

$$
t_{p}=\sqrt{h G / c^{5}}
$$

\section{4) Planck length $I_{p}$}

Planck length $l_{p}$ can be obtained from Lemma 7:

$$
l_{p}=t_{p} c
$$

Substituting formula (13) into formula (14) yields:

$$
l_{p}=\sqrt{h G / c^{3}}
$$

\subsection{Corollary}

Conversion is one of the core ideas of Cosmic Continuum Theory. The mutual transformation of mass, energy, and dark mass makes the universe colorful. However, these transformations are not arbitrary, but are determined by the existence boundaries of particles, quantum, and dark particles. With the mutual conversion between particles, quantum, and dark particles, space, time, and dark space also follow.

Corollary 1: When the mass of particles reaches Planck mass $m_{p}$, the particles are converted into quantum and the mass is converted into energy.

Proof: From (3), we can see that Planck mass $m_{p}$ is obtained by taking the speed as a variable to obtain the limit. According to Lemma 5, when the particle reaches the limit velocity $c$, it will turn into a quantum. According to the concept in [1], the energy body is a body composed of quantum, so when Planck mass is $m_{p}$, the mass has been transformed into energy. Q.E.D.

Corollary 2: When quantum energy reaches Planck energy $E_{p}$, quantum is transformed into dark particles and energy is converted into dark mass.

Proof: According to corollary 1, $m_{p} \equiv E_{p}$. From (3), we can see that Planck mass $m_{p}$ is obtained by taking the particle velocity as a variable and finding the limit. However, it is impossible for the particles to reach the limit velocity $c$, so that the mass of the particles cannot reach Plank mass $m_{p}$, and thus the quantum cannot reach Planck energy $E_{p}$. According to Lemma 4, the quantum frequency has a maximum value. As a limit value, if the quantum energy reaches Planck energy $E_{p}$, the frequency $v_{p}$ of the quantum must reach a maximum value of $v_{\max }$. According to Lemma 5 , when the quantum reaches the maximum frequency, it turns into a dark particle. According to the concept in [1], the dark mass is a body composed of dark particles. Therefore, when the quantum reaches a maximum frequency of $v_{\max }$, Planck energy $E_{p}$ is converted to a dark mass. Q.E.D.

Corollary 3: Planck mass $m_{p}$ is equivalent to Planck energy $E_{p}: m_{p} \equiv E_{p}$. 
Proof: From (7), we can see that Planck energy $E_{p}$ is obtained from Planck's mass $m_{p}$ based on Lemma 6, according to Lemma 6, then $m_{p} \equiv E_{p}$. Q.E.D.

Corollary 4: When the amount of time is less than or equal to Planck time $t_{p}$, the time is converted to dark space.

Proof: From (9), we can see that Planck time $t_{p}$ is derived from the Planck energy $E_{p}$ and the frequency $v_{p}$ of the quantum. However, to reach Planck energy $E_{p}$, the frequency $v_{p}$ of the quantum must reach a maximum of $v_{\max }$. According to corollary 2, the quantum is transformed into dark particles and the energy is converted into dark mass. According to the concept in [1], dark space is the existence dimension of dark masses. Therefore, when the amount of time is less than or equal to Planck time $t_{p}$, the time will be converted into a dark space. Q.E.D.

Corollary 5: When the amount of space is less than or equal to Planck length $l_{p}$, the space is converted to a dark space.

Proof: From (14), Planck length $l_{p}$ is the distance traveled at the speed of light in Planck time $t_{p}$. According to corollary 4 , when time is less than or equal to Planck time $t_{p}$, time has been converted to dark space. Therefore, when the amount of space is less than or equal to Planck length $l_{p}$, the space will also be converted into a dark space. Q.E.D.

Corollary 6: Planck time $t_{p}$ is equivalent to Planck length $l_{p}: t_{p} \equiv l_{p}$.

Proof: From (14), we can see that Planck length $l_{p}$ is obtained from Planck time $t_{p}$ based on Lemma 7. According to Lemma 7, then $t_{p} \equiv l_{p}$. Q.E.D.

The above 6 inferences fully demonstrate that Planck units do not have any logical contradictions and conflicts in the continuum of the universe and can perfectly express the physical boundaries and their transformation.

\section{Unity of Physical Dimensions}

The Planck unit system cleverly integrates the three fundamental constants of physics of Planck constant $h$, gravitational constant $G$, and speed of light $c$, and realized their dimensionless processing. Unfortunately, the physical significance of Planck units system is far from being fully reflected due to the inconsistency between the existing physical basic theories.

\subsection{New Understanding of the Benchmark Value of Planck Units}

The establishment of any physical dimension must be based on a certain benchmark. There are two kinds of benchmarks, one is the maximum, such as the speed of light $c$, the other is the minimum, such as the Planck constant $h$. There are many such benchmarks in the Planck units.

Corollary 7: The elementary unit of energy is $q_{\min }=h \mathrm{~J} . h$ is Planck constant.

Proof: According to Lemma 1 and Formula $E=h v$, where $E$ refers to the energy of the quantum and $v$ refers to the frequency of the quantum, it can be known that Planck constant $h$ is actually the energy of the unit frequency. This 
means that any energy body cannot be less than energy $h$, so Planck constant $h$ is the elementary unit of energy. Q.E.D.

Corollary 8: The elementary unit of mass is $m_{\min }=h / c^{2} \mathrm{~kg}$. $h$ is Planck constant, and $c$ is the speed of light.

According to Corollary 7, the elementary unit of energy is $q_{\min }=h$. According to Lemma 8 , the inertia of elementary particles and elementary quanta in an universe system is equivalent: $m_{\min } \equiv q_{\min }$. According to Lemma 6, the existence quantities of mass $m$ and energy $E=m c^{2}$ are equivalent: $m \equiv E$, so $h=m_{\min } c^{2}$. From this, we get: $m_{\min }=h / c^{2}$. Q.E.D.

Corollary 9: The elementary unit of time is $t_{\min }=t_{p} \mathrm{~s} . t_{p}$ is Planck time.

Proof: According to corollary 4, when the time is less than or equal to Planck time $t_{p}$, the time is converted to dark space. This shows that Planck time $t_{p}$ is the minimum value of time. That is, the elementary unit of time dimension $t_{\min }=t_{p}$. Q.E.D.

Corollary 10: The elementary unit of space is $s_{\min }=l_{p}$ m. $l_{p}$ is Planck length.

Proof: According to Corollary 5, when the length is less than or equal to Planck length $l_{p}$, the space is converted to dark space. This shows that Planck length $l_{p}$ is the minimum value of space. That is, the elementary unit of length $s_{\min }=l_{p}$. Q.E.D.

Corollary 11: The maximum value of frequency is $v_{\max }=1 / t_{p} \mathrm{~Hz} . t_{p}$ is Planck time.

Proof: According to corollary 9, the elementary unit of time is $t_{\min }=t_{p}, t_{p}$ is Planck time. This means that for any vibration cycle of the existing body $T$ : $T \geq t_{p}$, and frequency $v=1 / T$, so for any frequency: $v=1 / T \leq 1 / t_{p}$, ie. $v_{\max }=1 / t_{p}$. Q.E.D.

Corollary 12: The maximum value of quantum energy is $E_{\max }=E_{p}$ J. $E_{p}$ is Planck energy.

Proof: According to Corollary 11, the maximum value of frequency is $v_{\max }=1 / t_{p}, t_{p}$ is the Planck time. The energy of quantum is $E=h v, h$ is Planck constant, so $E_{\max }=h v_{\max }=h / t_{p}$. By (11), we can see that $E_{p}=h / t_{p}$, therefore $E_{\max }=E_{p}$. Q.E.D.

Corollary 13: The maximum value of particle mass is $m_{\max }=m_{p} \mathrm{~kg} \cdot m_{p}$ is Planck mass.

Proof: According to Lemma 10, The existences are coupled with each other by energy, And in the presence of different structural levels, by the corresponding quantum from the role of convergence. If there is a structure at the structural level of the quantum of $q$ connection, the corresponding structure of the particle $m$ is also the amount of $q: m \equiv q$. According to corollary 3 , Planck mass $m_{p}$ is equivalent to Planck energy $E_{p}: m_{p} \equiv E_{p}$. This shows that $m_{p}$ and $E_{p}$ are in the same structure level. According to corollary 12, The maximum value of quantum energy is $E_{\max }=E_{p}, E_{p}$ is Planck energy. That is, $E_{p}$ is at the maximum structural level. Therefore, $m_{p}$ is also at the structural level of 
maximum, that is, $m_{p}$ is the maximum value of particle mass: $m_{\max }=m_{p}$. Q.E.D.

Corollary 7-13 allows us to obtain the elementary unit of energy, the elementary unit of mass, the elementary unit of time, the elementary unit of space, the maximum of frequency, the maximum of quantum energy, and the maximum of particle mass 7 limit constants, this shows that the Cosmic Continuum Theory further develops the benchmark value of the Planck units.

\subsection{Equivalent Abstract Physical Dimension}

In the system of Planck units, the values of $h, G, c$ and Planck units are all equal to 1 , and the mass-energy equation is simplified to $E=m$. It looks a bit similar to Lemma 6: the existence quantities of mass $m$ and energy $E=m c^{2}$ are equivalent: $m \equiv E$. In reality, it is not. The former is a numerical simplification and is a non-dimensionalized process, represented by the "=" symbol. The latter is the physical equivalent of mass and energy, and it is the unity of physical dimensions, represented by the " $\equiv$ " symbol. In Lemma 6, the physical dimension of $m$ is kilogram, and the physical dimension of $E$ is Joule; When it satisfies $E=m c^{2}$, $m \equiv E \quad$ expresses: $m \mathrm{~kg} \equiv E \mathrm{~J}$.

Corollary 14: The physical dimensions of existence quantity can be unified on abstract physical dimensions.

Proof: According to Lemma 1, Lemma 8, the elementary unit of mass $m_{\min }$, the elementary unit of energy $q_{\min }$, and the elementary unit of dark quality $d_{\min }$ equivalence, which results in the existence of the elementary unit of quantity $e_{\min }: e_{\min } \equiv m_{\min } \equiv q_{\text {min }} \equiv d_{\text {min }}$.

Set $e_{\min }=1 \mathrm{JX}$, then:

$$
\begin{gathered}
1 \mathrm{JX} \equiv m_{\min }=h / c^{2}(\mathrm{~kg}) \\
1 \mathrm{JX} \equiv q_{\min }=h(\mathrm{~J}) \\
1 \mathrm{JX} \equiv d_{\min } \text { Unit }
\end{gathered}
$$

Due to the fact that there is no international standard unit for the amount of dark mass, it is temporarily replaced by "Unit". According to Lemma 11, there are only particle, quantum, and dark particle three basic forms, thus we have unified the physical dimension of existence quantity to an abstract physical dimension "JX":

$$
e_{\min }=1 \mathrm{JX} \equiv h / c^{2} \mathrm{~kg} \equiv h \mathrm{~J} \equiv d_{\min } \text { Unit }
$$

So the physical dimensions of existence quantity can be unified on abstract physical dimensions. Q.E.D.

Corollary 15: The physical dimensions of dimension quantity can be unified on abstract physical dimension.

Proof: According to Lemma 9, the elementary unit of space $s_{\min }$, the elementary unit of time $t_{\min }$, and the elementary unit of dark space $g_{\min }$ are equivalent, so as to produce the elementary unit of dimension $w_{\min }: w_{\min } \equiv s_{\min } \equiv t_{\min } \equiv g_{\min }$.

Set $w_{\min }=1 \mathrm{XJ}$, then: 


$$
\begin{gathered}
1 \mathrm{XJ} \equiv t_{\min }=t_{p}=\sqrt{h G / c^{5}}(\mathrm{~s}) \\
1 \mathrm{XJ} \equiv s_{\min }=l_{p}=\sqrt{h G / c^{3}}(\mathrm{~m}) \\
1 \mathrm{XJ} \equiv g_{\text {min }} \text { Unit }
\end{gathered}
$$

Similarly, there is currently no international standard unit for the amount of dark space, it is temporarily replaced by "Unit". According to Lemma 11, there are only three basic forms of particle, quantum, and dark particle. According to Concept 4 , Concept 5 , and Concept 6 , there are also only three basic dimensional forms: space, time, and dark space. Therefore, we have unified the physical dimension of dimension quantity to an abstract physical dimension "XJ":

$$
w_{\min }=1 \mathrm{XJ} \equiv \sqrt{h G / c^{5}} \mathrm{~s} \equiv \sqrt{h G / c^{3}} \mathrm{~m} \equiv d_{\min } \text { Unit }
$$

So the physical dimensions of dimension quantity can be unified on abstract physical dimension. Q.E.D.

Corollary 16: All changes in the universe can be equivalent on abstract physical dimensions.

Proof: All changes in the universe are changes in the quantity and dimension of existence. According to Corollary 14, the physical dimensions of existence quantity can be unified on abstract physical dimensions; and according to Corollary 15 , the physical dimensions of dimension quantity can be unified on abstract physical dimension. According to Lemma 8 and Lemma 9, the unity of physical dimensions is based on physical equivalence, so all changes in the universe can be equivalent on abstract physical dimensions. Q.E.D.

Obviously, this physical dimensions system is exactly the same as the Planck Units System. However, the two physical dimensions "JX" and "XJ" are essentially different from our existing physical dimensions. Existing physical dimensions are specific physical dimensions, including Planck units. There is no connection between different specific physical dimensions. But the "JX" and "XJ" are abstract physical dimensions. The function of an abstract physical dimension is to achieve the unity of specific physical dimensions.

\subsection{A New Interpretation of Gravitational Redshift}

The following is an attempt to solve the gravitational redshift problem by applying the equivalent unified scheme of physical dimensions proposed in this paper.

We know that the energy of a photon $E$ is:

$$
E=h v
$$

$h$ is the Planck constant and $v$ is the photon frequency. According to Lemma 6, The existence quantities of mass $m$ and energy $E=m c^{2}$ are equivalent: $m \equiv E$. Therefore:

$$
E=h v \equiv m c^{2}
$$

$c$ is the speed of light. So we can get:

$$
m \equiv h v / c^{2}
$$


According to Lemma 12, it can be seen that the photon escapes from a certain celestial body $M$ to a distant place of $R$, and the change of gravitational potential energy $E_{p}$ is:

$$
E_{p}=-G M m / R
$$

$G$ is the gravitational constant. According to corollary 14, the physical dimensions of existence quantity can be unified on abstract physical dimensions. Substituting (26) into (27) gives:

$$
E_{p}=-G M h v / R c^{2}
$$

The amount of change in the gravitational potential energy of a photon is the amount of energy change of this photon. According to the formula (24), if the photon frequency change amount is $v_{p}$, then:

$$
v_{p}=E_{p} / h
$$

Substituting (28) into (29):

$$
v_{p}=-G M v / R c^{2}
$$

Deform (30) to get:

$$
v_{p} / v=-G M / R c^{2}
$$

The result is consistent with the gravitational redshift formula derived by general relativity. This shows that the introduction of the concept of relativistic mass is equivalent to the unification of physical dimensions.

\section{Conclusion}

The new fundamental constants of physics often represent a new basic theory of physics, and the logical unity of basic physical constants is essentially the unity of physics. The Planck system of units profoundly reveals the physical boundary problem, but it has always been constrained by inconsistencies between the basic theories of physics. In Cosmic Continuum Theory, Planck Units System completely escapes the incompatibility of the physical basis. The axiomatization of Planck Units System enables the logical unity of fundamental constants of physics that represent different physical foundations to be realized. In particular, based on Planck unit, 7 limit constants were deduced, giving a unified benchmark for physical dimensions. The introduction of abstract physical dimensions "JX" and "XJ" to achieve unity of physical dimensions will sweep away another barrier for the unification of physics.

\section{Conflicts of Interest}

I declare that there is no competing Interest.

\section{References}

[1] Wang, X.J. (2018) Journal of Modern Physics, 9, 1250-1270. https://doi.org/10.4236/jmp.2018.96074 
[2] Wang, X.J. and Wu, J.X. (1992) The Unity Theory. Haitian Publishing House, Shenzhen.

[3] Wang, X.J. and Wu, J.X. (2001) Crack to the Puzzle of Scientific Unity. Hunan Science \& Technology Press, Changsha.

[4] Planck, M. (1899) Über irreversible Strahlungsvorgänge. Sitzungsberichte der Königlich Preußischen Akademie der Wissenschaften zu Berlin, 5: 440-480. (pp. 478-480 contain the first appearance of the Planck base units other than the Planck charge, and of Planck's constant, which Planck denoted by b)

[5] Wesson, P.S. (1980) Space Science Reviews, 27, 109-153.

[6] Duff, M., Okun, L.B. and Veneziano, G. (2002) Journal of High Energy Physics, 3, 23. https://doi.org/10.1088/1126-6708/2002/03/023

[7] Ashtekar, A. (2013) Loop Quantum Gravity and the The Planck Regime of Cosmology, arXiv:1303.4989.

[8] Gonzalez-Mestres, L. (2014) EPJ Web of Conferences, 71, 00063.

[9] Post, E.J. (1982) Foundations of Physics, 12, 169-195. https://doi.org/10.1007/BF00736847

[10] Robinett, R.W. (2015) American Journal of Physics, 83, 353-361. https://doi.org/10.1119/1.4902882

[11] Fischer, J. and Ullrich, J. (2016) Nature Physics, 12, 4-7. https://doi.org/10.1038/nphys3612

[12] Kisak, P.F. (2015) Planck Units: The Fundamental Scale of Cosmology. CreateSpace Independent Pub, Colorado Springs.

[13] CODATA Value: Newtonian Constant of Gravitation. The NIST Reference on Constants, Units, and Uncertainty. US National Institute of Standards and Technology. June 2015. Retrieved 2015-09-25. 2014 CODATA Recommended Values.

[14] Staff. Birth of the Universe. University of Oregon. Retrieved September 24, 2016. (Discusses "Planck time" and "Planck era" at the very beginning of the Universe)

[15] Barrow, J.D. (2002) The Constants of Nature; From Alpha to Omega-The Numbers that Encode the Deepest Secrets of the Universe. Pantheon Books, New York.

[16] Barrow, J.D. and Tipler, F.J. (1988) The Anthropic Cosmological Principle. Oxford University Press, Oxford.

[17] Dirac, P.A.M. (1938) Proceedings of the Royal Society A, 165, 199-208. https://doi.org/10.1098/rspa.1938.0053

[18] Tomilin, K.A. (1999) Natural Systems of Units: To the Centenary Anniversary of the Planck System, 287-296.

[19] Pavšic, M. (2001) The Landscape of Theoretical Physics: A Global View. Kluwer Academic, Dordrecht, 347-352. https://arxiv.org/abs/gr-qc/0610061

[20] Davies, P.C., Davis, T.M. and Lineweaver, C.H. (2002) Nature, 418, 602-603.

[21] Nieto, J., Poupaud, F. and Soler, J. (2001) Archive for Rational Mechanics and Analysis, 158, 29-59. https://doi.org/10.1007/s002050100139 